\title{
Editorial
}

\section{Acknowledgement to Reviewers of Membranes in 2013}

Membranes Editorial Office, MDPI AG, Klybeckstrasse 64, CH-4057 Basel, Switzerland

Published: 24 February 2014

The editors of Membranes would like to express their sincere gratitude to the following reviewers for assessing manuscripts in 2013:

\begin{tabular}{|c|c|c|}
\hline Aili, David & Gallucci, Fausto & Maranas, Janna K. \\
\hline Albertin, Luca & Giannici, Francesco & Marcos, Bernard \\
\hline Barredo-Damas, S. & Gilron, Jack L. & Mather, Glenn C. \\
\hline Basile, Angelo & Granville, Anthony M. & Mazzei, Rosalinda \\
\hline Bellona, Christopher & Groth, Andrew & Menkhaus, Todd J. \\
\hline Besser, Ronald S. & Hägg, May-Britt & Miyoshi, Shogo \\
\hline Borsi, Iacopo & Herzing, Andrew & Moore, Robert B. \\
\hline Bracewell, Daniel G. & Hesampour, Mehrdad & Naessens, Wouter \\
\hline Bredesen, Rune & Hoek, Eric M. V. & Nikkola, Juha \\
\hline Budd, Peter & Hong, Liang & Nopens, Ingmar \\
\hline Cazorla-Amorós, Diego & Janiak, Christoph & Nørgaard, C. F. \\
\hline Chang, Feng-Chih & Karabelas, A. J. & Ortiz, Inmaculada \\
\hline Chen, Jinhua & Kern, Wolfgang & Pant, Deepak \\
\hline Cheng, Liao-Ping & Khatib, Sheima Jatib & Paraskeva, Christakis A \\
\hline Cho, Jinwoo & Kim, In-Chul & Park, Sang-Eon \\
\hline Chu, Benjamin & Kim, Jeonghwan & Ramon, Guy \\
\hline Chu, Peter Po-Jen & Kuznicki, Steven M. & Rodrigues, Alírio E. \\
\hline Chung, Tai-Shung & Kyu, Thein & Romanos, George E. \\
\hline Ciardelli, Francesco & Lawler, Jenny & Rustad, James R. \\
\hline Cohen, Yoram & Lee, Kew-Ho & Samhaber, W. M. \\
\hline Coronas, Joaquín & Liné, Alain & Schiffman, Jessica D. \\
\hline Deeke, Alexandra & Linkous, Clovis A. & Schröder, Uwe \\
\hline Deng, Baolin & Lu, Xiaoyun & Serra, Jose M. \\
\hline Escobar, Isabel C. & Luis, Patricia & Servili, Maurizio \\
\hline Fong, Hao & Ma, Guang-Hui & Sleutels, Tom H. J. A. \\
\hline Fullerton-Shirey, Susan K. & Malaisamy, Ramamoorthy & Smart, Simon \\
\hline
\end{tabular}


Teli, Shivanand B.

Tseng, Hui-Hsin

Van Rijn, Cees Jm

Winnubst, Louis
Wu, Rome-Ming

Yamaki, Junichi

Yamaki, Tetsuya

Yampolskii, Yuri
Zhang, Liangmin

(C) 2014 by the authors; licensee MDPI, Basel, Switzerland. This article is an open access article distributed under the terms and conditions of the Creative Commons Attribution license (http://creativecommons.org/licenses/by/3.0/). 